\title{
Short communication: Possible mechanism for inhibiting the formation of polymers originated from 5-hydroxymethyl-2-furaldehyde by sulfite groups in the dairy thermal process
}

\author{
Yong-Guang Guan, ${ }^{*}$ Si-Ming Zhu, ${ }^{*}$ Shu-Juan $\mathrm{Yu},{ }^{*}+\ddagger^{1}$ Xian-Bing $\mathrm{Xu},{ }^{*}$ and Li-Cai Zhu§ \\ ${ }^{*}$ College of Light Industry and Food Sciences, South China University of Technology, Guangzhou 510640, China \\ †State Key Laboratory of Pulp and Paper Engineering, Guangzhou 510640, China \\ ¥Guangdong Province Key Laboratory for Green Processing of Natural Products and Product Safety, Guangzhou 510640, China \\ $\S$ School of Chemistry and Environment, South China Normal University, Guangzhou 510631, China
}

\begin{abstract}
5-Hydroxymethyl-2-furaldehyde can undergo polymerization to form high-molecular weight molecules via the Maillard reaction during dairy thermal treatment. In this study, the effect of sulfite group on polymer formation, especially in inhibiting the formation of highmolecular weight polymers has been described. Results showed that the sulfite group significantly inhibited the increase of polymer molecular weight via prevention of the polymerization of 5-hydroxymethyl-2-furaldehyde. The formation of an intermolecular dimer based on the glucose molecule through Schiff base cyclization can lead to a competitive reaction with 1,2-enolization to reduce 5-hydroxymethyl-2-furaldehyde formation, which might be another factor in reducing the formation of high-molecular weight polymers.
\end{abstract}

Key words: 5-hydroxymethyl-2-furaldehyde, polymer, sulfite group

\section{Short Communication}

The Maillard reaction is one of the most important and complex processes during dairy thermal treatment (Sithole et al., 2005; Lillard et al., 2009; Dattatreya et al., 2010; Corzo-Martínez et al., 2011; Guan et al., 2012), which always produces 5-hydroxymethyl-2-furaldehyde (HMF) based on sugar decomposition (Hodge, 1953). The presence of HMF has been confirmed as a main factor leading to polymer formation (Yaylayan and Kaminsky, 1998), resulting in browning and lowering of dairy quality. Recently, much research has detailed the decomposition of HMF to form polymers. For instance, Chambel et al. (1998) used HMF as a precursor to synthesize ether dehydrated by $2 \mathrm{HMF}$ molecules. The same dimer was also identified earlier by Popoff and Theander (1976). Previous studies (Nikolov and

Received August 13, 2012.

Accepted February 10, 2013.

${ }^{1}$ Corresponding author: shujuanyu8@gmail.com
Yaylayan, 2011) suggested that vinylogous aldol addition could be considered a possible mechanism for the generation of dimeric structures based on aldol condensation of $2 \mathrm{HMF}$ molecules. Thus, it can be said that HMF may be one of the precursors during polymer formation (the proposed polymeric structure is shown in Figure 1).

A sulfite group has been confirmed to inhibit the initial phases of the Maillard reaction (Guan et al., 2011). However, whether a sulfite group could inhibit the formation of polymers in the advanced phases of the Maillard reaction is still unclear. Hence, understanding the interaction of HMF with sulfite groups during dairy thermal treatment may provide further insight into its polymerization reactivity mechanism. The aim of this research was to confirm the effects of sulfite groups on the inhibition of polymer formation originated from HMF.

Methanol of chromatographic grade was purchased from Merck KGaA (Darmstadt, Germany). 5-Hydroxymethyl-2-furaldehyde, glucose, glycine, and ammonium sulfite were purchased from Sigma-Aldrich (St. Louis, $\mathrm{MO}$ ).

Samples were prepared using a high-pressure vessel (4522M; Parr Instrument Co., Moline, IL). Reaction system A was $15 \mathrm{~g}$ of glucose and $3.0 \mathrm{~g}$ of glycine mixed in $15 \mathrm{~mL}$ of double-distilled (dd) water. Reaction system B was $15 \mathrm{~g}$ of glucose and $3.4 \mathrm{~g}$ of ammonium sulfite mixed in $15 \mathrm{~mL}$ of dd water. All mixtures were heated at $120^{\circ} \mathrm{C}$ for different times and then cooled to room temperature by immediately placing on iced water to stop the reaction. Ion resin (Zhengguang ZG C107; Zhengguang Resin Co. Ltd., Hangzhou, China) was used for stable intermediate product (SIP) separation. The pretreatment method was as follows: $1 \mathrm{~L}$ of ZG C107 ion-exchange resin $\left(\mathrm{Na}^{+}\right.$form) was washed with $5 \% \mathrm{NaOH}$ solution $(1 \mathrm{~L})$ and water $(2$ L), respectively, and then eluted with $4 \% \mathrm{HCl}(1 \mathrm{~L})$ to transform the $\mathrm{Na}^{+}$form to the $\mathrm{H}^{+}$form. After the ion-exchange resin was abundantly transformed to the $\mathrm{H}^{+}$form, dd water was used to elute the resin until the 


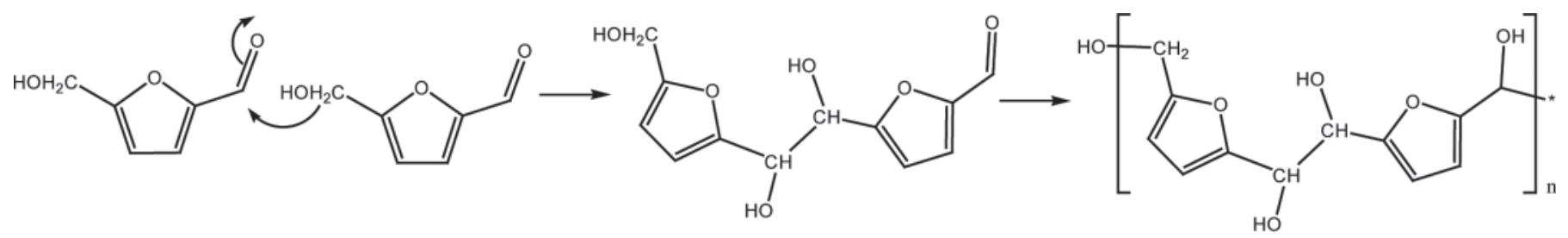

Compound 1

Figure 1. Proposed polymeric structure originated from 5-hydroxymethyl-2-furaldehyde (HMF; Nikolov and Yaylayan, 2011).

$\mathrm{pH}$ of the eluent was above 6.5 . The reaction mixture was dissolved in an ethanol/water mixture $(70+30$, $\mathrm{vol} / \mathrm{vol} ; 1 \mathrm{~L})$ and then passed through a column $(3.5$ $\times 50 \mathrm{~cm}$; Maolin Glass Apparatus Ltd., Guangzhou, China) filled with the pretreated resin. The resin was washed with an ethanol/water mixture $(70+30, \mathrm{vol} /$ vol; $5 \mathrm{~L})$, water $(1.5 \mathrm{~L})$, and ammonium hydroxide $(0.2 \mathrm{~mol} / \mathrm{L})$ successively, and $100-\mathrm{mL}$ fractions of the eluent were collected. Each fraction was tested using HPLC-tandem mass spectrometry (HPLC-MS/MS) and high-performance anion-exchange chromatography (HPAEC)-electrochemical detector (ECD) analyses (Guan et al., 2012). N-(1-Deoxy-D-glucose-1-yl)-glycine (the Amadori compound) and HMF were separated and isolated in reaction system A, whereas HMF and some white amorphous powder were obtained in reaction system B. For further separation, $N$-(1-deoxy-Dglucose-1-yl)-glycine, HMF and the unidentified white amorphous powder were isolated by a preparative liquid chromatograph using a C18 $(10-\mu \mathrm{m})$ column $(25$ $\times 400 \mathrm{~mm}$, i.d. $\times$ length) with a UV detector (Lisui; Suzhou Lisui Technology Co. Ltd., Suzhou, China). The gradient used was between $100 \%$ water to $100 \%$ methanol for $120 \mathrm{~min}$, with a flow rate of $15 \mathrm{~mL} / \mathrm{min}$. The monitor was set at 215 and $254 \mathrm{~nm}$. The eluted SIP were concentrated in vacuum at $45^{\circ} \mathrm{C}$ and subsequently used for electrospray ionization-tandem mass spectrometry (ESI-MS/MS) and GC-MS analysis. $N$-(1Deoxy-D-glucose-1-yl)-glycine was obtained as a white crystallization powder. White amorphous powder was obtained and named as compound 2. 5-Hydroxymethyl2 -furaldehyde was also obtained as a yellow oily liquid. $N$-(1-Deoxy-D-glucose-1-yl)-glycine and compound 2 were hygroscopic and were, therefore, stored in a desiccator at $4^{\circ} \mathrm{C}$ before further testing in $72 \mathrm{~h}$.

The ESI-MS/MS assay was carried out as described earlier (Mastovska and Lehotay, 2006), with slight modifications. The ESI-MS/MS analysis was used for further identification of the SIP. In the current experiment, mass spectrometry was carried out in an LCQ Fleet mass spectrometer (Thermo Fisher, San Jose, $\mathrm{CA}$ ), with an ESI source (positive and negative mode). A $1-\mu \mathrm{L}$ volume of the isolated compounds $(1 \mathrm{mg} / \mathrm{mL}$ in water) was directly injected into the mass spectrometer in the $m / z$ range of 50 to 1,000 .

The presence of HMF was determined using a Varian 3800 series gas chromatograph with a mass spectrometric (electron impact mode) detector 4000 (Varian Inc., Santa Clara, CA). Before GC-MS analysis, samples were freeze dried, and 10-mg freeze-dried samples were dissolved in $200 \mathrm{~mL}$ of methanol and subsequently filtered through a 0.45 - $\mu \mathrm{m}$-pore-size filter paper (Millipore Corp., Billerica, MA). An Agilent HP-INNOWAX column $(30 \mathrm{~m} \times 0.25-\mathrm{mm}$ i.d., with film thickness of $0.25 \mu \mathrm{m}$ ) was used. The injector temperature of the gas chromatograph was $250^{\circ} \mathrm{C}$. The initial temperature was $40^{\circ} \mathrm{C}$ and held for $4 \mathrm{~min}$, and then increased from 40 to $50^{\circ} \mathrm{C}$ at a rate of $10^{\circ} \mathrm{C} / \mathrm{min}$. Thereafter, the temperature was increased from 50 to $120^{\circ} \mathrm{C}$ at a rate of $6^{\circ} \mathrm{C} / \mathrm{min}$ and then increased from 120 to $220^{\circ} \mathrm{C}$ at a rate of $10^{\circ} \mathrm{C} /$ min and held for $7 \mathrm{~min}$. The injection volume was $5 \mu \mathrm{L}$. The carrier gas was helium, flowing at a rate of $1 \mathrm{~mL} /$ min. Mass spectra of the compounds were compared with spectra from the National Institute of Standards and Technology NIST05 spectral library (Guan et al., 2011).

Nuclear magnetic resonance (NMR) analysis was done at $125 \mathrm{MHz}$ for ${ }^{13} \mathrm{C}$ NMR in a Bruker Avance 600 instrument (Bruker Co., Faellanden, Switzerland). The data were processed by the MestReNova program (Mestrelab Research, Santiago de Compostela, Spain). The NMR data of $N$-(1-deoxy-D-glucose-1-yl)-glycine and compound 2 were obtained in $\mathrm{D}_{2} \mathrm{O}$.

The molecular weight (MW) distribution profiles of the polymer were assessed by an HPLC-diode array detector (DAD) system using a TSKgel G4000PWXL $7.8 \times 300-\mathrm{mm}$ column (Tosoh Corp., Tokyo, Japan). All peaks were tested at $284 \mathrm{~nm}$. Data acquisition and chromatographic analysis were driven by the Empower software program (Waters, Milford, MA). The injection volume was $20 \mu \mathrm{L}$ (sterilized through $0.45-\mu \mathrm{m}$ filters), and elution was carried out with Milli-Q water (Millipore Corp.) at a flow rate of $0.5 \mathrm{~mL} / \mathrm{min}$. The column temperature was maintained at $30^{\circ} \mathrm{C}$. In this experiment, all data were values of relative dextran molecular mass, calculated based on the following formula: 
GUAN ET AL.
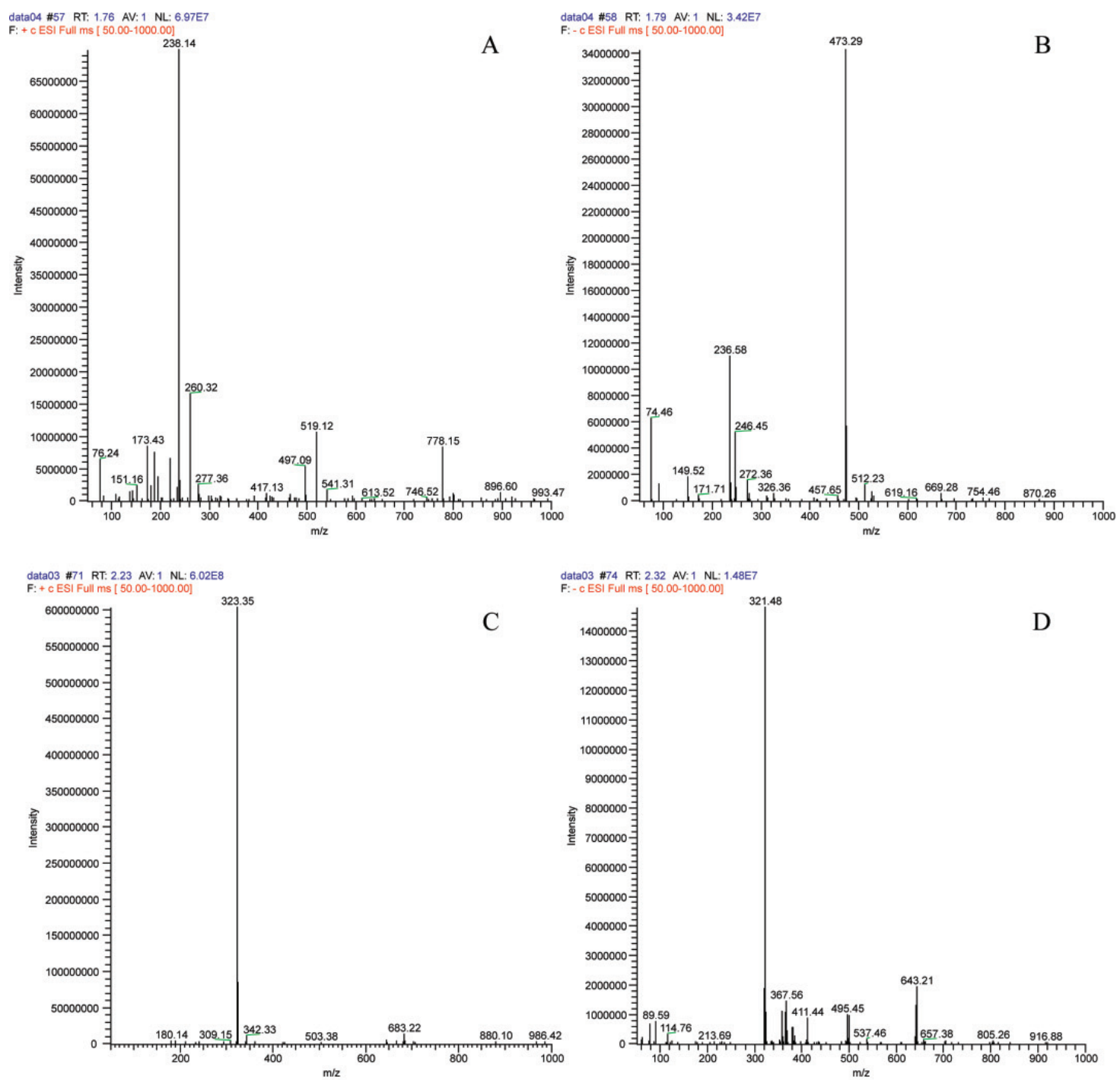

data03 \#74 RT: 2.32 AV:1 NL: $1.48 E 7$
F:- c ESI Full ms [50.00-1000.00]
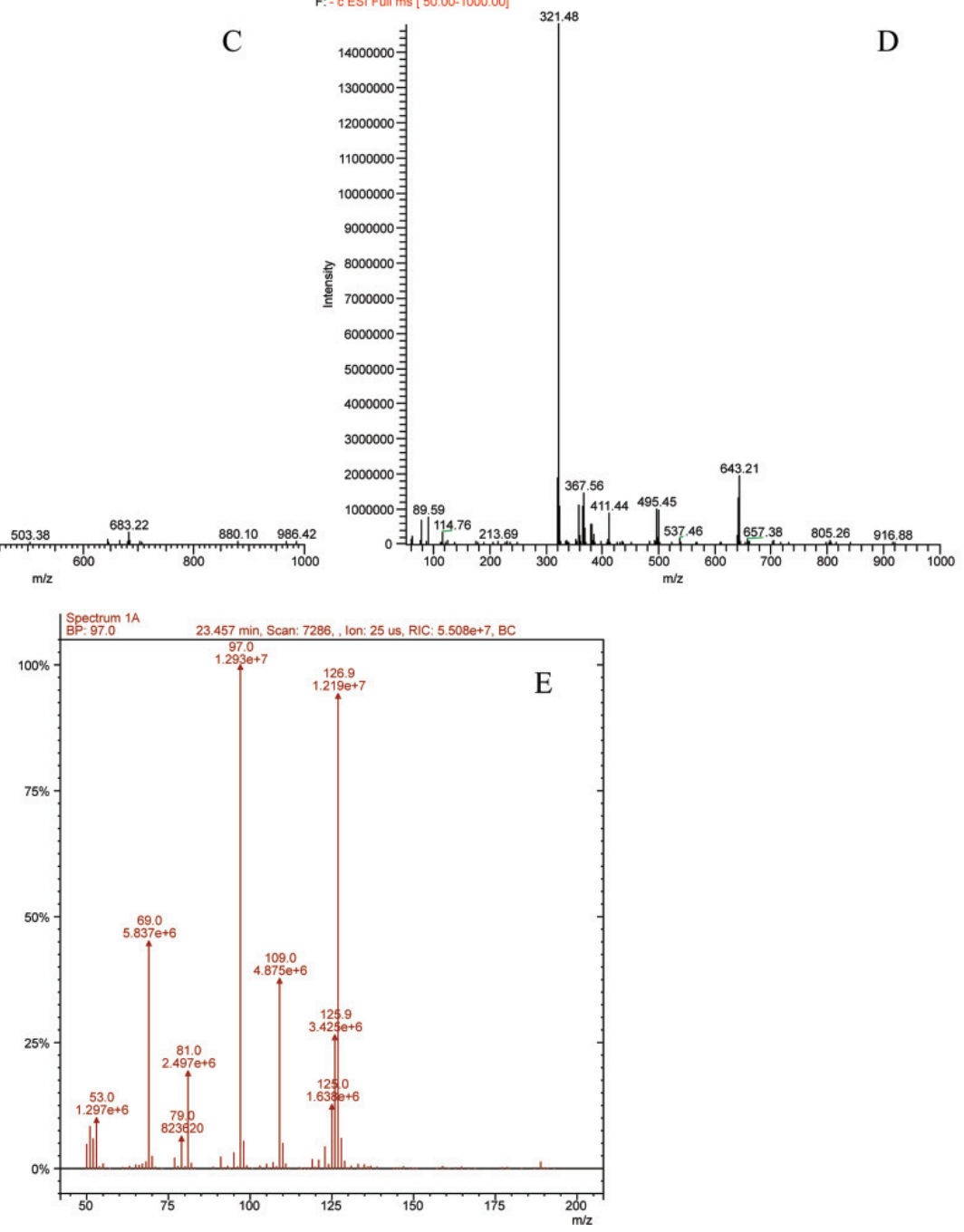

Figure 2. Electrospray ionization-tandem mass spectrometry (ESI-MS/MS) information for $N$-(1-deoxy-D-glucose-1-yl)-glycine in positive (A) and negative (B) modes and compound 2 in positive (C) and negative (D) modes, and electron ionization-mass spectrometry (EI-MS) of 5-hydroxymethyl-2-furaldehyde (HMF; E). Color version available in the online PDF. 


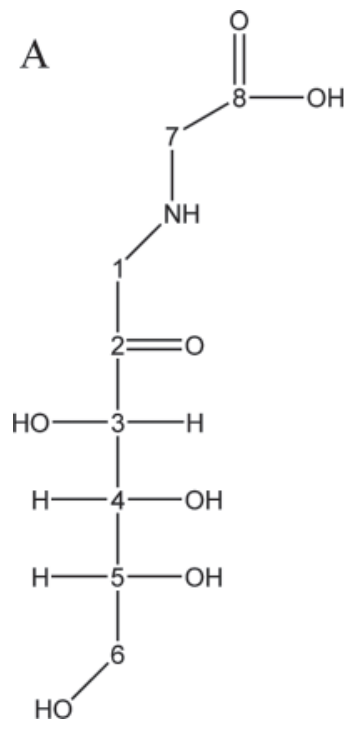

Figure 3. The nuclear magnetic resonance (NMR) data of $N-(1-$ deoxy-D-glucose-1-yl)-glycine (A) and compound 2 (B).

$$
\lg \mathrm{MW}=-0.2796 t+9.3041\left(\mathrm{R}^{2}=0.99\right),
$$

where lg MW is the common logarithm of the value of relative dextran molecular mass of sample, and $t$ is the retention time.

All experiments were carried out in triplicate. Means and standard deviations of the data were calculated for each treatment. Analysis of variance was carried out to determine any significant differences $(P<0.05)$ among the applied treatments by the SPSS package (SPSS 10.0 for Windows; SPSS Inc., Chicago, IL).

Two SIP [i.e., $N$-(1-deoxy-D-glucose-1-yl)-glycine and HMF] were tested in the glucose-glycine system, whereas compound 2 (an intermolecular dimer based on the glucose molecule via a Schiff base) and HMF were obtained in the glucose-ammonium sulfite system. The ESI-MS/MS and electron ionization-mass spectrometry (EI-MS) information is shown in Figure 2. The structures of $N$-(1-deoxy-D-glucose-1-yl)-glycine and compound 2 are shown in Figure 3. In the positive mode of MS/MS analysis, the Amadori compound $N$-(1-deoxy-D-glucose-1-yl)-glycine showed the most abundant peak $[\mathrm{M}+\mathrm{H}]^{+}$at $m / z=238.1$. Apart from $[\mathrm{M}+\mathrm{H}]^{+}$, the fragment $\left[\mathrm{M}+\mathrm{H}-2 \mathrm{H}_{2} \mathrm{O}-\mathrm{CO}\right]^{+}$at $\mathrm{m} / z=$ 173.4, $[\mathrm{Gly}+\mathrm{H}]^{+}$at $m / z=76.2$, and the adduct ion $[\mathrm{M}$ $+\mathrm{Na}^{+}$at $m / z=260.3$ were detected. In the negative mode, the adduct ion $[2 \mathrm{M}-\mathrm{H}]^{-}$at $\mathrm{m} / \mathrm{z}=473.3$ showed the most abundant peak; $[\mathrm{M}-\mathrm{H}]^{-}$at $m / z=236.6$, [M$\left.\mathrm{H}-2 \mathrm{H}_{2} \mathrm{O}-\mathrm{CO}\right]^{-}$at $m / z=171.7$, and $[\mathrm{Gly}-\mathrm{H}]^{-}$at $\mathrm{m} / z$ $=74.5$ were also detected. The data of ${ }^{13} \mathrm{C} \mathrm{NMR}$ for $\mathrm{N}$-(1-deoxy-D-glucose-1-yl)-glycine are as follows: ${ }^{13} \mathrm{C}$ NMR (125 MHz, $\left.\mathrm{D}_{2} \mathrm{O}\right): \mathrm{C} 1: 53.89$ ppm; C2: 215.82
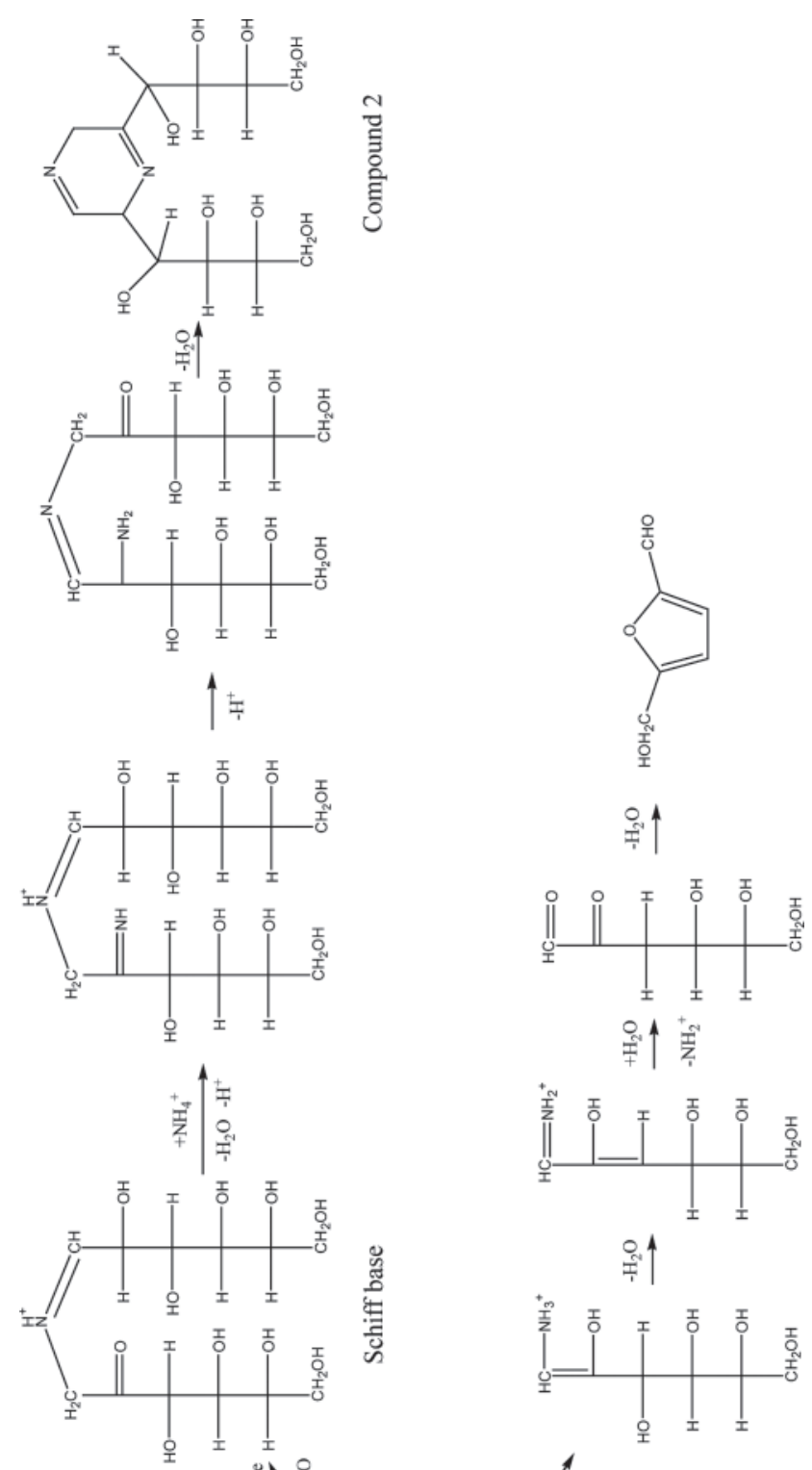


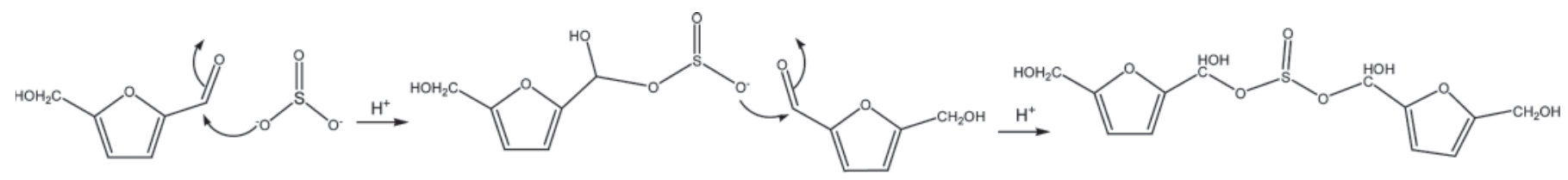

Compound 3

Figure 5. Proposed formation path of compound 3.

ppm; C3: 70.80 ppm; C4: 77.29 ppm; C5: 65.88 ppm; C6: $61.87 \mathrm{ppm}$; C7: $50.75 \mathrm{ppm}$; C8: $171.22 \mathrm{ppm}$. As for compound $2,[\mathrm{M}+\mathrm{H}]^{+}$at $m / z=323.4$ was tested in the positive model, whereas $[\mathrm{M}-\mathrm{H}]^{-}$at $m / z=321.5$ and $[2 \mathrm{M}-\mathrm{H}]^{-}$at $m / z=643.2$ were observed in the negative mode. The MS/MS of the $[\mathrm{M}+\mathrm{H}]^{+}$and $[\mathrm{M}-\mathrm{H}]^{-}$was acquired at compensation voltage $=20 \mathrm{~V}$. The data of ${ }^{13} \mathrm{C}$ NMR for compound 2 are as follows: ${ }^{13} \mathrm{C}$ NMR (125 $\left.\mathrm{MHz}, \mathrm{D}_{2} \mathrm{O}\right): \mathrm{C} 1: \delta 166.26 \mathrm{ppm}, \mathrm{C} 2: \delta 161.59 \mathrm{ppm}, \mathrm{C} 3$ : $\delta 62.45 \mathrm{ppm}, \mathrm{C} 4: \delta 72.09 \mathrm{ppm}, \mathrm{C} 5: \delta 69.78 \mathrm{ppm}, \mathrm{C} 6$ : $\delta 73.90 \mathrm{ppm}, \mathrm{C} 7: \delta 73.55 \mathrm{ppm}, \mathrm{C} 8: \delta 63.46 \mathrm{ppm}, \mathrm{C} 9: \delta$ 77.73 ppm, C10: $\delta 74.80$ ppm, C11: $\delta 72.81 \mathrm{ppm}, \mathrm{C} 12$ : $\delta 63.49$ ppm. 5-Hydroxymethyl-2-furaldehyde has a formyl group and a methylol group, which make it weak for soft ionization. Therefore, electron impact is more suitable to show a significant response of HMF mass spectrometry information as $m / z=126.9,97.0,81.0$, 69.0, and 53.0. Formation of $N$-(1-deoxy-D-glucose1-yl)-glycine and HMF in the glucose-glycine system was proposed in earlier research (Davidek et al., 2002, 2005). $\quad N$-(1-Deoxy-D-glucose-1-yl)-glycine was established by the degradation of the Schiff base, which was formed in the initial phase of the Maillard reaction via Amadori rearrangement of $N$-glycosylamines; the latter was obtained by condensation of glucose and glycine. 5-Hydroxymethyl-2-furaldehyde, as the main SIP, has been proposed by the degradation of $N$-(1-deoxy-Dglucose-1-yl)-glycine via 1,2-enolization. However, 2 main SIP (i.e., compound 2 and HMF) were obtained in the glucose-ammonium sulfite system, which indicated that except the formation of HMF by the degradation of $N$-(1-deoxy-D-glucose-1-yl)-ammonia via 1,2-enolization, other polymerization pathways such as $N$-(1-deoxy-D-glucose-1-yl)-ammonia and glucose that react to form the Schiff base might exist. This Schiff base then loses one molecule of water and condenses with $\mathrm{NH}_{4}{ }^{+}$, in a chain reaction, losing $\mathrm{H}^{+}$and undergoing Amadori rearrangement to generate the precursor of compound 2 (Figure 4). The formation of compound 2 might reduce the accumulation of HMF, which can serve as a precursor for the construction of polymeric structures during the Maillard reaction.

Molecular weight is an important parameter that reflects the degree of polymerization of a polymer. A
high-MW polymer (>600,000 Da) was observed even when the reaction time was only $1 \mathrm{~h}$ in the glucoseglycine system. However, only a low-MW polymer $(<10,000 \mathrm{Da})$ was obtained in the glucose-ammonium sulfite reaction system even after $5 \mathrm{~h}$ at $120^{\circ} \mathrm{C}$.

The formation of polymers in the glucose-glycine system is an instantaneous reaction, which should be carried out mainly through the polymerization of HMF and its derivative (Nikolov and Yaylayan, 2011). However, in the glucose-ammonium sulfite system, polymerization was significantly inhibited. One of the possible explanations could be detailed as follows: vinylogous aldol addition between $2 \mathrm{HMF}$ is carried out to form a HMF dimer, which can undergo further polymerization at the hydroxymethyl terminal or carbonyl terminal of the molecule, or continue polymerization with other HMF molecules through a series of condensations, and finally generate a polymer (Nikolov and Yaylayan, 2011). However, in the presence of a sulfite group, the nucleophilic reaction between the sulfite group and the carbon atom of the carbonyl group of 2 molecules of HMF is carried out, and reduces the formation of an HMF dimer. A proposed structure suggested as compound 3 (Figure 5) should be formed by the reaction of 2 HMF molecules, with 1 molecule of the sulfite group, which consumes the carbonyl group of HMF, and leads to the inhibition of polymerization (Nikolov and Yaylayan, 2011). Therefore, sulfite group inhibition of the polymerization of HMF to form a polymer might be one of the explanations of the data obtained in this experiment.

A sulfite group inhibits the formation of polymer via the nucleophilic reaction between the sulfite group and the carbon atom of the carbonyl group, and inhibits the formation of an HMF dimer. The formation of compound 2, as a intermolecular dimer based on the glucose molecule, leads to a competitive reaction with 1,2-enolization to reduce HMF, and decreases the formation of high-MW polymers.

\section{ACKNOWLEDGMENTS}

All authors acknowledge the National Science Foundation of China (Beijing; no. 31071564), the Key 
Foundation of Guangdong Province of China (no. 2009A020700007), and the National Science Foundation of China (no. 31271889) for financial support. All authors appreciate Enoch's help in language expression.

\section{REFERENCES}

Chambel, P., M. B. Oliveira, P. B. Andrade, J. O. Fernandes, R. M. Seabra, and M. A. Ferreira. 1998. Identification of 5,5'-oxy-dimethylene-bis (2-furaldehyde) by thermal decomposition of 5-hydroxymethyl-2-furfuraldehyde. Food Chem. 63:473-477.

Corzo-Martínez, M., A. C. Soria, M. Villamiel, A. Olano, F. M. Harte, and F. J. Moreno. 2011. Effect of glycation on sodium caseinatestabilized emulsions obtained by ultrasound. J. Dairy Sci. 94:5158.

Dattatreya, A., W. Lee, and S. A. Rankin. 2010. Presence of galactose and glucose promotes browning of sweet whey powder. J. Dairy Sci. 93:2354-2357.

Davidek, T., N. Clety, S. Aubin, and I. Blank. 2002. Degradation of the Amadori compound $N$-(1-deoxy-D-fructos-1-yl)glycine in aqueous model systems. J. Agric. Food Chem. 50:5472-5479.

Davidek, T., K. Kraehenbuehl, S. Devaud, F. Robert, and I. Blank 2005. Analysis of Amadori compounds by high-performance cation exchange chromatography coupled to tandem mass spectrometry. Anal. Chem. 77:140-147.

Guan, Y.-G., S.-L. Wang, S.-J. Yu, S.-M. Yu, and Z.-G. Zhao. 2012. Changes in the initial stages of a glucose-proline Maillard reaction model systems influences the dairy quality during thermal processing. J. Dairy Sci. 95:590-601.

Guan, Y.-G., S.-M. Zhu, S.-J. Yu, X.-B. Xu, and W.-H. Shi. 2011. $\mathrm{SO}_{3}{ }^{2-}$ effects the 5-hydroxymethyl-2-furaldehyde content in ammonium sulphite-glucose solutions. Int. J. Food Sci. Technol. 46:1007-1013.

Hodge, J. E. 1953. Dehydrated foods: Chemistry of browning reactions in model systems. J. Agric. Food Chem. 1:1928-1943.

Lillard, J. S., D. A. Clare, and C. R. Daubert. 2009. Glycosylation and expanded utility of a modified whey protein ingredient via carbohydrate conjugation at low pH. J. Dairy Sci. 92:35-48.

Mastovska, K., and S. J. Lehotay. 2006. Rapid sample preparation method for LC-MS/MS or GC-MS analysis of acrylamide in various food matrices. J. Agric. Food Chem. 54:7001-7008.

Nikolov, P. Y., and V. A. Yaylayan. 2011. Thermal decomposition of 5-(hydroxymethyl)-2-furaldehyde (HMF) and its further transformations in the presence of glycine. J. Agric. Food Chem. 59:10104-10113.

Popoff, T., and O. Theander. 1976. Formation of aromatic compounds from carbohydrates. Part III. Reaction of D-glucose and D-fructose in slightly acidic, aqueous solution. Acta Chem. Scand. A 30:397-402.

Sithole, R., M. R. McDaniel, and L. M. Goddik. 2005. Rate of Maillard browning in sweet whey powder. J. Dairy Sci. 88:1636-1645.

Yaylayan, V. A., and E. Kaminsky. 1998. Isolation and structural analysis of Maillard polymers: Caramel and melanoidin formation in glycine/glucose model system. Food Chem. 63:25-31. 